J. Korean Math. Soc. 49 (2012), No. 6, pp. 1139-1161

http://dx.doi.org/10.4134/JKMS.2012.49.6.1139

\title{
SUMS OF $\left(p^{r}+1\right)$-TH POWERS \\ IN THE POLYNOMIAL RING $\mathbb{F}_{p^{m}}[T]$
}

\author{
Mireille CAR
}

\begin{abstract}
Let $p$ be an odd prime number and let $F$ be a finite field with $p^{m}$ elements. We study representations and strict representations of polynomials $M \in F[T]$ by sums of $\left(p^{r}+1\right)$-th powers. A representation

$$
M=M_{1}^{k}+\cdots+M_{s}^{k}
$$

of $M \in F[T]$ as a sum of $k$-th powers of polynomials is strict if $k \operatorname{deg} M_{i}<$ $k+\operatorname{deg} M$.
\end{abstract}

\section{Introduction}

Let $F$ be a finite field of characteristic $p$ with $p^{m}$ elements and let $k>1$ be an integer. The similarity between the $\operatorname{ring} \mathbb{Z}$ of rational integers and the polynomial ring $F[T]$ had led to investigations of an analogue of the Waring problem for $F[T]$ (See [2], [6], [11], [14], [17], [19], [20], [21], [22] for general exponent $k$ or [4], [5], [8], [9], [10] for some particular exponents). Roughly speaking, Waring's problem over $F[T]$ is that of the representation of polynomials $M \in F[T]$ as sums

$$
M=M_{1}^{k}+\cdots+M_{s}^{k}
$$

with $M_{1}, \ldots, M_{s} \in F[T]$. Some obstructions to that may occur which led to considering Waring's problem over the subring $\mathcal{S}(F, k)$ formed by the polynomials of $F[T]$ which are sums of $k$-th powers. Two variants of Waring's problem over $\mathcal{S}(F, k)$ have been considered. The unrestricted Waring's problem is the problem of proving the existence of an integer $w=w\left(p^{m}, k\right)$, with the property that whenever $M \in \mathcal{S}(F, k)$ and $s \geq w\left(p^{m}, k\right)$, the equation (1.1) is solvable. This problem is close to the so called easy Waring's problem for $\mathbb{Z}$ ([17], [18], [19], [20]). In order to have an analogue for the non easy Waring problem, the degree conditions

$$
\operatorname{deg} M_{i} \leq n
$$

Received November 26, 2010; Revised July 8, 2012.

2010 Mathematics Subject Classification. Primary 11T55; Secondary 11R58.

Key words and phrases. finite fields, polynomials, Waring's problem. 
are required with $n$ defined by the condition

$$
k(n-1)<\operatorname{deg} M \leq k n .
$$

With such degree conditions, the representation (1.1) is strict in opposition to representations without degree conditions. For the strict Waring's problem, analogues to the classical Waring's numbers $g_{\mathbb{N}}(k)$ and $G_{\mathbb{N}}(k)$ have been defined as follows. Let $g\left(p^{m}, k\right)$, respectively, $G\left(p^{m}, k\right)$, denote the least integer $s$, if it exists, such that every polynomial $M \in \mathcal{S}(F, k)$, respectively, every polynomial $M \in \mathcal{S}(F, k)$ of sufficiently large degree, may be written as a sum (1.1) satisfying the degree conditions (1.2) and (1.3). Otherwise, $g\left(p^{m}, k\right)$, respectively, $G\left(p^{m}, k\right)$ is equal to $\infty$. This notation is possible since these numbers depend only on $p^{m}$ and $k$. Waring's problem consists of determining or, at least, bounding the numbers $g\left(p^{m}, k\right)$ and $G\left(p^{m}, k\right)$.

Gallardo's method introduced in [8] and performed in [5] to deal with Waring's problem for cubes was generalized in [2] and [11] where bounds for $g\left(p^{m}, k\right)$ and $G\left(p^{m}, k\right)$ were established when $p^{m}$ and $k$ satisfy some conditions. For instance, Theorem 1.2 in [2] and Theorem 1.4 in [11] require that every $a \in F$ is a sum of $k$-th powers and $p^{m}>k$. Theorem 1.3 in [2] gives a bound for the numbers $g\left(p^{m}, k\right)$ in the case where $p>k$ or in the case $k=h p^{\nu}-1<p^{m}$ for some positive integers $\nu$ and $h \leq p$.

The case of the exponent $k=p^{r}+1$ is not covered by these theorems. The object of this paper is the study of Waring's problem in the case where $k=p^{r}+1$ for odd $p$. It can be seen as a generalisation of [4] where sums of biquadrates over a field of characteristic 3 were studied. The easier case $p=2$ has been studied in [3].

Some notations and definitions are necessary before stating the main results proved in this work.

The set $\mathcal{S}(F, k)$ and the numbers $g\left(p^{m}, k\right)$ and $G\left(p^{m}, k\right)$ are not sufficient to describe every possible case. Proposition 4.5 in [2] and Proposition 3.7 in [3] give examples of polynomials in $\mathcal{S}(F, k)$ which are not strict sums of $k$-th powers. Thus, we introduce new parameters.

Let $\mathcal{S}^{\times}(F, k)$ denote the set of polynomials in $F[T]$ which are strict sums of $k$-th powers. Let $g^{\times}\left(p^{m}, k\right)$, respectively $G^{\times}\left(p^{m}, k\right)$, denote the least integer $s$, if it exists, such that every polynomial $M \in \mathcal{S}^{\times}(F, k)$ respectively, every polynomial $M \in \mathcal{S}^{\times}(F, k)$ of sufficiently large degree, may be written as a strict sum

$$
M=M_{1}^{k}+\cdots+M_{s}^{k}
$$

From now on, $F$ is a finite field with $p^{m}$ elements. The main results proved in this work are summarized in the following theorems.

Theorem 1.1. Let $k=p^{r}+1$, where $p$ is an odd prime number and $r$ a positive integer. 
(1) If $m / \operatorname{gcd}(m, r) \geq 3$, then the set $\mathcal{S}(F, k)$ is equal to the whole ring $F[T]$ and

where

$$
\mathcal{S}^{\times}(F, k)=\mathcal{A}_{\infty} \cup\left(\bigcup_{N=0}^{k-3} \mathcal{A}_{N}\right),
$$

$$
\mathcal{A}_{\infty}=\{A \in F[T] \mid \operatorname{deg} A>k(k-3)\}, \quad \mathcal{A}_{0}=F,
$$

and for $N=1, \ldots, k-3$,

$$
\mathcal{A}_{N}=\left\{A \in F[T] \mid A=\sum_{n=0}^{N} \sum_{i=0}^{N} x_{n, i} T^{i+n p^{r}}\right\}
$$

with $x_{n, i} \in F$.

(2) If $m$ divides $r$,

$\mathcal{S}^{\times}(F, k)=\mathcal{S}(F, k)=\left\{A \in F[T] \mid A^{p^{r}}-A \equiv 0 \quad\left(\bmod \quad T^{p^{2 r}}-T\right)\right\}$.

(3) If $m / \operatorname{gcd}(m, r)=2$,

$$
\mathcal{S}(F, k)=\left\{A \in F[T] \mid A^{p^{r}}-A \equiv 0 \quad\left(\bmod T^{p^{2 r}}-T\right)\right\},
$$

and $\mathcal{S}^{\times}(F, k)$ is the set formed by the $A \in \mathcal{S}(F, k)$ such that, either $\operatorname{deg} A$ is not multiple of $k$, or $\operatorname{deg} A$ is multiple of $k$ and the leading coefficient of $A$ is in the subfield of $F$ of order $p^{\operatorname{gcd}(m, r)}$.

This theorem is a consequence of Corollary 3.3, Proposition 5.1, and Corollaries 5.4 and 5.6 below.

Theorem 1.2. Let $k=p^{r}+1$, where $p$ is an odd prime number and $r$ a positive integer.

(1) (a) If $m / \operatorname{gcd}(m, r) \geq 3, m / \operatorname{gcd}(m, r) \neq 4$, and if $p^{m}$ is congruent to 1 modulo 4,

$$
\begin{aligned}
G\left(p^{m}, k\right)= & G^{\times}\left(p^{m}, k\right) \leq \min \left(\frac{\log k}{\log (k /(k-1))}+5,2 k+3\right) ; \\
g^{\times}\left(p^{m}, k\right) & \leq 5 k-4 .
\end{aligned}
$$

(b) If $m / \operatorname{gcd}(m, r) \geq 3$, and if $p^{m}$ is congruent to 3 modulo 4,

$$
\begin{aligned}
& G\left(p^{m}, k\right)= G^{\times}\left(p^{m}, k\right) \leq \min \left(\frac{\log k}{\log (k /(k-1))}+6,3 k+3\right) ; \\
& g^{\times}\left(p^{m}, k\right) \leq 6 k-4 .
\end{aligned}
$$

(c) If $m / \operatorname{gcd}(m, r)=4$,

$$
\begin{aligned}
G\left(p^{m}, k\right)= & G^{\times}\left(p^{m}, k\right) \leq \min \left(\frac{\log k}{\log (k /(k-1))}+6,2 k+4\right) ; \\
g^{\times}\left(p^{m}, k\right) & \leq 6 k-6 .
\end{aligned}
$$

(d) If $m / \operatorname{gcd}(m, r) \geq 3$, then $g\left(p^{m}, k\right)=\infty$. 
(2) (a) If $m$ divides $r$ and if $p^{m}$ is congruent to 1 modulo 4,

$$
\begin{aligned}
G\left(p^{m}, k\right) & =G^{\times}\left(p^{m}, k\right) \leq 2 k ; \\
g\left(p^{m}, k\right) & =g^{\times}\left(p^{m}, k\right) \leq 3 k-6 .
\end{aligned}
$$

(b) If $m$ divides $r$ and if $p^{m}$ is congruent to 3 modulo 4,

$$
\begin{gathered}
G\left(p^{m}, k\right)=G^{\times}\left(p^{m}, k\right) \leq 3 k ; \\
g\left(p^{m}, k\right)=g^{\times}\left(p^{m}, k\right) \leq 3 k .
\end{gathered}
$$

(3) If $m / \operatorname{gcd}(m, r)=2$,

$$
\begin{gathered}
G\left(p^{m}, k\right)=g\left(p^{m}, k\right)=\infty, \\
G^{\times}\left(p^{m}, k\right) \leq g^{\times}\left(p^{m}, k\right) \leq 2 k .
\end{gathered}
$$

This theorem is a consequence of Corollaries 3.5, 5.4 and 5.6 below. It shows that the analogy with the rational integers does not work completely since the following bounds hold for large exponents $k$ :

$$
G_{\mathbb{N}}(k) \leq k(\log k+\log (\log k)+O(1)) ;
$$

see [23] and

$$
2^{k}+\left[(3 / 2)^{k}\right]-2 \leq g_{\mathbb{N}}(k) \leq 2^{k}+\left[(3 / 2)^{k}\right]+\left[(4 / 3)^{k}\right]-2
$$

(see [7], [12, Chap. 21], [23]).

With the necessary adaptations, the proof follows the method used in [3] where we dealt with the case of characteristic 2 . We omit the proofs in [3].

Let $v\left(p^{m}, k\right)$ denote the least integer $v$, if it exists, such that $T$ may be written as a sum $\left(a_{1} T+b_{1}\right)^{k}+\cdots+\left(a_{v} T+b_{v}\right)^{k}$ with $a_{i}, b_{i} \in F$. Otherwise, let $v\left(p^{m}, k\right)=\infty$. If $v\left(p^{m}, k\right)$ is finite, every $P \in F[T]$ may be written as a sum

$$
P=\left(a_{1} P+b_{1}\right)^{k}+\cdots+\left(a_{v(F, k)} P+b_{v(F, k)}\right)^{k}
$$

so that $\mathcal{S}(F, k)=F[T]$ and $F$ is a $k$-Waring field.

As in the case $p=2$, it is possible to compute the exact value of $v\left(p^{m}, p^{r}+1\right)$. This improves a theorem of Paley [15].

The paper is organized as follows. In order to get the exact value of $v\left(p^{m}, k\right)$ we have to prove that some algebraic equations have solutions in $F$. This is done in Section 2. In Section 3, we compute the numbers $v\left(p^{m}, k\right)$. This yields a characterization of the fields $F$ for which the equality $\mathcal{S}(F, k)=F[T]$ holds. Some bounds for the Waring numbers $G\left(p^{m}, k\right)$ follow. In Section 4 , we prove some key identities and we classify strict sums of degree $\leq k(k-2)$. In Section 5 , we describe a descent process and we conclude the proof. We shall use two types of numbering. Pairs $(X . Y)$ will be used to number formulae occurring in definitions, propositions and theorems, single numbers $(z)$ will be used for formulae only used in the course of a proof.

If every $a \in F$ is a sum of $k$-th powers, the field $F$ is called a Waring field for the exponent $k$ or briefly, a $k$-Waring field. If $F$ is a $k$-Waring field, let $\ell\left(p^{m}, k\right)$ denote the least integer $\ell$ such that every element of $F$ is a sum of $\ell$ 
$k$-th powers. We shall denote by $\lambda\left(p^{m}, k\right)$ the least integer $s$ such that -1 is a sum of $s k$-th powers. We write $\Delta\left(p^{m}, k\right)$ for $\operatorname{gcd}\left(p^{m}-1, k\right)$.

We fix an algebraic closure $\bar{F}$ of the field $F$. For a positive integer $n$, we denote by $\mathbb{F}_{p^{n}}$ the subfield of $\bar{F}$ with $p^{n}$ elements, so that $F=\mathbb{F}_{p^{m}}$. The proofs will often use the following facts:

- the field $F$ contains exactly $\Delta\left(p^{m}, k\right)=\operatorname{gcd}\left(p^{m}-1, k\right)=\operatorname{gcd}\left(p^{m}-\right.$ $\left.1, p^{r}+1\right) k$-th roots of 1 ;

- a $k$-th power in $F$ is a $\operatorname{gcd}\left(p^{m}-1, k\right)$-th power.

We introduce the notations

$$
\begin{gathered}
Q=p^{r}=k-1, \quad q=p^{\operatorname{gcd}(m, r)}, \\
d=\operatorname{gcd}(m, r)
\end{gathered}
$$

so that

$$
q=p^{d}
$$

If $x$ is a real number, we denote by $[x]$ its integral part and by $\lceil x\rceil$ its ceiling, that is the least integer $n \geq x$.

\section{Sums of $k$-th powers in the finite field $F$}

Since a $k$-th power in $F$ is a $\operatorname{gcd}\left(p^{m}-1, k\right)$-th power, we begin this section by computing $\Delta=\Delta\left(p^{m}, k\right)$. We continue by a study of a sum of characters which will be useful to compute numbers of solutions of some equations.

The following proposition completes Lemma 4 in [15]. It is a special case of exercise 125 in De Koninck and Mercier's book. See [13, exercise 125, p. 23, solution p. 125].

Proposition 2.1. One has

$$
\operatorname{gcd}\left(p^{m}-1, p^{r}-1\right)=p^{d}-1 .
$$

The greatest common divisor of $p^{m}-1$ and $p^{r}+1$ is an even number. Moreover, $\operatorname{gcd}\left(p^{m}-1, p^{r}+1\right) \neq 2$ if and only if $m / d$ is even and, in that case,

$$
\operatorname{gcd}\left(p^{m}-1, p^{r}+1\right)=p^{d}+1 \text {. }
$$

\subsection{The systems $\mathcal{E}(u, v, a, b)$ and $\mathcal{S}(a, b, c)$}

Lemma 2.2. Let $(u, v) \in F^{2}$ be such that $u v \neq 0$ and $u^{Q^{2}-1} \neq v^{Q^{2}-1}$. For every ordered pair $(a, b) \in F^{2}$, the system $\mathcal{E}(u, v, a, b)$ :

$$
\left\{\begin{array}{l}
a=u^{Q} x+v^{Q} y, \\
b=u x^{Q}+v y^{Q},
\end{array}\right.
$$

admits a unique solution in $F^{2}$.

Proof. Immediate. 
Lemma 2.3. Let $(a, b, c) \in F^{3}$. Then, the system $\mathcal{S}(a, b, c)$ :

$$
\left\{\begin{array}{l}
a=x^{2}+y^{2}+z^{2} \\
b=x \xi+y \eta+z \zeta \\
c=\xi^{2}+\eta^{2}+\zeta^{2}
\end{array}\right.
$$

has a solution $(x, y, z, \xi, \eta, \zeta)$ in $F^{6}$.

Proof. Serre's theorem asserts that with the exceptions of polynomials of degree 3 and 4 in the case $q=3$, every polynomial in $F[T]$ is a strict sum of 3 squares ([6, Theorem 1.14, p. 7]). Applied to $P=a T^{2}+2 b T+c$, Serre's theorem gives the existence of $(x, y, z, \xi, \eta, \zeta) \in F^{6}$ such that

$$
a T^{2}+2 b T+c=(x T+\xi)^{2}+(y T+\eta)^{2}+(z T+\zeta)^{2},
$$

so that $(x, y, z, \xi, \eta, \zeta)$ is a solution of $\mathcal{S}(a, b, c)$.

When $m / d$ is odd, $\operatorname{gcd}\left(2^{m}-1, k\right)=2$, and the set of $k$-th powers in $F$ is the set of squares, so that the numbers $\nu_{i}(a)$ of representations of $a \in F$ as sums of $i k$-th powers are well known (see e.g. [1]). We compute the numbers $\nu_{i}(a)$ in the case where $m / d$ is even. For that we introduce some character sums.

\subsection{Sums of characters}

In this subsection, we suppose that $m / d$ is even, so that $\mathbb{F}_{q^{2}} \subset F$. From Proposition 2.1, the set of $k$-th powers in $F$, resp. in $\mathbb{F}_{q^{2}}$ is the set of $(q+1)$-th powers in $F$, resp. in $\mathbb{F}_{q^{2}}$. Let

$$
n=m / 2 d \text {. }
$$

Let $\theta$ be a generator of the cyclic group $\mathbb{F}_{q^{2}}^{\times}$and let

$$
\alpha=\theta^{(q+1) / 2} .
$$

Let tr: $F \mapsto \mathbb{F}_{p}$ be the absolute trace on $F$ and let $\psi$ be the character of the additive group of $F$ defined by

$$
\psi(x)=\exp \left(\frac{2 \pi i \operatorname{tr}(x)}{p}\right) .
$$

Then $\psi$ is not trivial. For $t \in F$ let

$$
f(t)=\sum_{x \in F} \psi\left(t x^{q+1}\right) .
$$

Let $B$ denote the set of non-zero $k$-th powers in $F$ or, equivalently, the set of non-zero $(q+1)$-th powers in $F$.

Proposition 2.4. (1) If $u \in \mathbb{F}_{q^{2}}$, then $u^{q+1} \in \mathbb{F}_{q}$.

(2) For every $u \in \mathbb{F}_{q}$, there is $v \in \mathbb{F}_{q^{2}}$ such that $u=v^{q+1}$.

(3) One has

$$
f(0)=p^{m} .
$$

(4) Let $t \in F^{\times}$. 
(a) If $t \in \alpha B$, then

$$
f(t)=f(\alpha)=(-q)^{n+1}
$$

(b) If $t \notin \alpha B$, then

$$
q f(t)+f(\alpha)=0 .
$$

Proof. (1) If $u \in \mathbb{F}_{q^{2}}$, then $\left(u^{q+1}\right)^{q-1}=u^{q^{2}-1}=1$, so that $u^{q+1} \in \mathbb{F}_{q}$.

(2) Since $\theta$ generates $\mathbb{F}_{q^{2}}^{\times}$, the cyclic group $\mathbb{F}_{q}^{\times}$is generated by $\theta^{q+1}$, so that every $u \in F_{q}^{\times}$is a power of $\theta^{q+1}$.

(3) Obvious.

(4) It is a generalization of $[4$, Proposition 2.2]. See the proof of [3, Proposition 2.4(i)] and [3, Proposition 2.5] for the proof of (2.10); see the proof of [3, Proposition 2.4(iii)] for the proof of (2.11).

\subsection{Sums of $k$-th powers in $F$}

Let $i$ be a positive integer. For $a \in F$, let $\nu_{i}(a)$ denote the number of solutions $\left(x_{1}, \ldots, x_{i}\right) \in F^{i}$ of the equation

$$
a=x_{1}^{k}+\cdots+x_{i}^{k} .
$$

Proposition 2.5. Suppose $\mathrm{m} / \mathrm{d}$ odd.

- If $q \equiv 1(\bmod 4)$, then,

$$
\begin{aligned}
& \nu_{2}(0)=2 p^{m}-1, \\
& \nu_{3}(0)=p^{2 m}
\end{aligned}
$$

and for $a \in F^{\times}$, one has

$$
\begin{gathered}
\nu_{2}(a)=p^{m}-1, \\
\nu_{3}(a)=\left\{\begin{array}{lll}
p^{2 m}+p^{m} & \text { if } & a \in B, \\
p^{2 m}-p^{m} & \text { if } & a \notin B .
\end{array}\right.
\end{gathered}
$$

- If $q \equiv 3(\bmod 4)$, then,

$$
\begin{gathered}
\nu_{2}(0)=1, \\
\nu_{3}(0)=p^{2 m}
\end{gathered}
$$

and for $a \in F^{\times}$, one has

$$
\begin{gathered}
\nu_{2}(a)=p^{m}+1, \\
\nu_{3}(a)=\left\{\begin{array}{lll}
p^{2 m}-p^{m} & \text { if } & a \in B, \\
p^{2 m}+p^{m} & \text { if } & a \notin B .
\end{array}\right.
\end{gathered}
$$

Proof. Observe that $a \in F$ is a $k$-th power if and only if $a$ is a square. Apply the well-known results on sums of squares in a finite field, [1, exercise 5, pp. 175$176]$. 
Proposition 2.6. Suppose $m / d$ even. Then,

$$
\begin{gathered}
\nu_{2}(0)=(q+1) p^{m}-q, \\
\nu_{3}(0)=p^{2 m}+f(\alpha)(q-1)\left(p^{m}-1\right)
\end{gathered}
$$

and for $a \in F^{\times}$, one has

$$
\begin{gathered}
\nu_{1}(a)=\left\{\begin{array}{lll}
q+1 & \text { if } & a \in B, \\
0 & \text { if } \quad a \notin B,
\end{array}\right. \\
\nu_{2}(a)=p^{m}-q+(q-1) f(a \alpha), \\
\nu_{3}(a)=p^{2 m}-p^{m}+p^{m} \nu_{1}(a)-(q-1) f(\alpha)+(q-1) f(\alpha) f(a \alpha) .
\end{gathered}
$$

Proof. Similar to that of Proposition 2.7 in [3].

Proposition 2.7. $\quad$ - $F$ is a Waring field for the exponent $k=p^{r}+1$ if and only if $\frac{m}{d} \neq 2$.

- If $\frac{m}{d} \neq 2$, then $\ell\left(p^{m}, k\right)=2$.

Proof. From Proposition 2.1, if $m / d$ is odd, then $\Delta\left(p^{m}, k\right)=2$. From [2, Proposition 3.1], $F$ is a $k$-Waring field with $\ell\left(p^{m}, k\right)=2$. Now, suppose $\frac{m}{d}$ even. Set $m=2 n d$. From Proposition 2.1, $\Delta\left(p^{m}, k\right)=1+p^{d}$. Since $\Delta\left(p^{m}, k\right)>1$, we have $\ell\left(2^{m}, k\right) \geq 2$. We prove that, with the exception $n=1, F$ is a $k$-Waring field with $\ell\left(p^{m}, k\right) \leq 2$. Let $a \in F$ be different from a $k$-th power. From Proposition 2.6, then Proposition 2.4,

$\nu_{2}(a)=p^{m}-q+(q-1) f(a \alpha) \geq p^{m}-q-(q-1) p^{m / 2}=q^{2 n}-q-q^{n+1}+q^{n}$.

If $n>1$, then $\nu_{2}(a)>0$ and $a$ is the sum of two $k$-th powers. Thus, if $a \in F$, either $a$ is a $k$-th power or $a$ is a sum of two $k$-th powers. Hence, $\ell\left(p^{m}, k\right)=\ell(F, k) \leq 2$ (Note that Small had already established this bound in the case where $m>4 r,[16])$.

Remark 2.8. We have $\lambda\left(p^{m}, k\right)=1$ if and only if $p^{m}$ is congruent to 1 modulo 4.

Proof. If $\lambda\left(p^{m}, k\right)=1$, then -1 is a $k$-th power in $F$, so that -1 is a square in $F$. Now, we suppose that -1 is a square in $F$. Firstly, we suppose $m / d$ odd. From Proposition 2.1, the set of $k$-th powers in $F$ is the set of squares in $F$, so that -1 is a $k$-th power in $F$. Secondly, suppose $m / d$ even. Then, $\mathbb{F}_{q^{2}} \subset F$. Since $\theta$ generates the cyclic group $\mathbb{F}_{q^{2}}$, we have

$$
-1=\theta^{\left(q^{2}-1\right) / 2}=\left(\theta^{(q-1) / 2}\right)^{q+1}
$$

with $\theta \in \mathbb{F}_{q^{2}} \subset F$. From Proposition 2.1, the set of $k$-th powers in $F$ is the set of $(q+1)$-th powers in $F$. Therefore -1 is a $k$-th power in $F$. 
Proposition 2.9. For $a \in F$, let $N_{3}(a)$ denote the number of $(x, y, z) \in F^{3}$ such that

$(\mathcal{F}(a)) \quad \begin{cases}x^{k}+y^{k}+z^{k}=a, & \left(e_{1}\right) \\ x y \neq 0, & \left(e_{2}\right) \\ x^{Q^{2}-1} \neq y^{Q^{2}-1} & \left(e_{3}\right)\end{cases}$

- Suppose $m / d$ even. Then,

$$
N_{3}(0)=p^{2 m}-p^{m}\left(q^{3}+1\right)+q^{3}+(q-1)\left(p^{m}-1\right) f(\alpha)
$$

and for $a \in F^{\times}$, one has

$N_{3}(a)= \begin{cases}p^{2 m}+p^{m}\left(q^{3}-3 q^{2}-1\right)+2 q^{3}-(q-1)\left(q^{2}-q+1\right) f(\alpha) & \text { if } a \in B, \\ p^{2 m}-p^{m}\left(2 q^{2}-2 q+1\right)+q^{3}-q^{2}+(q-1)(q-2) f(\alpha) & \text { if } a \notin B,\end{cases}$ where $\alpha$ is as in (2.6) and $f$ as in (2.8).

- Suppose $m / d$ odd. Then,

$$
N_{3}(0)=\left(p^{m}-1\right)\left(p^{m}-q\right)
$$

and for $a \in F^{\times}$,

$$
N_{3}(a)=\left\{\begin{array}{lll}
\left(p^{m}-2\right)\left(p^{m}-q\right) & \text { if } & a \in B \\
p^{m}\left(p^{m}-q\right) & \text { if } & a \notin B .
\end{array}\right.
$$

Proof. The proof is a generalization of the proof of Proposition 2.6 in [4]. In the case of [4], $p=3$ and $k=4$, so that the proof only needs to distinguish two cases depending on the parity of $m$. In the present general setting we have to distinguish different cases according to whether or not $F$ contains $\mathbb{F}_{q^{2}}$, and according to whether or not -1 is a $k$-th power in $F$.

Corollary 2.10. Let $a \in F$.

(1) If $a \neq 0$ and $m / d \geq 3$, or if $a=0$ and $m / d \geq 3$ with $m / d \neq 4$, then $(\mathcal{F}(a))$ has solutions in $F^{3}$.

(2) If $m / d \leq 2$, for any $a \in F,(\mathcal{F}(a))$ has no solutions in $F^{3}$.

(3) Suppose $m=4 d$. Then $(\mathcal{F}(0))$ has no solutions in $F^{3}$. Let $a \in F$. Then, there exists $(x, y, z, u) \in F^{4}$ such that

$(\mathcal{G}(a))$

$$
\begin{cases}x^{k}+y^{k}+z^{k}+u^{k}=a, & \left(e_{1}\right) \\ x y \neq 0, & \left(e_{2}\right) \\ x^{Q^{2}-1} \neq y^{Q^{2}-1} & \left(e_{3}\right)\end{cases}
$$

Proof. If $m / d \leq 2$, then $F \subset \mathbb{F}_{q^{2}}$, so that $\left(e_{3}\right)$ is not satisfied in $F$. This proves the second claim. We prove the other claims.

(A) Suppose $m / d$ even, say $m=2 n d$ with $n>1$. From Proposition 2.9,

$$
N_{3}(0)=q^{4 n}-q^{2 n}\left(q^{3}+1\right)+q^{3}+(q-1)\left(q^{2 n}-1\right) f(\alpha) .
$$

By $(2.10)$,

$$
N_{3}(0)=q^{4 n}-q^{2 n}\left(q^{3}+1\right)+q^{3}+(q-1)\left(q^{2 n}-1\right)(-q)^{n+1} .
$$


If $n>2$, then $N_{3}(0)>0$, so that $(\mathcal{F}(0))$ has a solution. If $n=2$, then $N_{3}(0)=0$, so that $(\mathcal{F}(0))$ has no solutions. Let $a \in B$. From Propositions 2.9 and 2.4

$$
\begin{aligned}
N_{3}(a) & \geq p^{2 m}+p^{m}\left(q^{3}-3 q^{2}-1\right)+2 q^{3}-(q-1)\left(q^{2}-q+1\right) q p^{m / 2} \\
& >p^{2 m}+p^{m}\left(q^{3}-3 q^{2}-1-q(q-1)\left(q^{2}-q+1\right)\right) \\
& =p^{2 m}-p^{m}\left(q^{4}-3 q^{3}+5 q^{2}+q-1\right) \\
& >q^{4 n}-q^{2 n+4} \geq 0 .
\end{aligned}
$$

Thus, $(\mathcal{F}(a))$ has a solution. Let $a \in F^{\times} \backslash B$. From Propositions 2.4 and 2.9,

$$
\begin{aligned}
N_{3}(a) & \geq p^{2 m}-p^{m}\left(2 q^{2}-2 q+1\right)+q^{3}-q^{2}-(q-1)(q-2) q p^{m / 2} \\
& >p^{2 m}-p^{m}\left(q^{3}-q^{2}+1\right) \\
& >p^{2 m}-p^{m} q^{3}=q^{4 n}-q^{2 n+3}>0 .
\end{aligned}
$$

If $n \geq 2$, then $N_{3}(a)>0$. Thus, $(\mathcal{F}(a))$ has a solution. Suppose $n=2$. If $a \neq 0$, for every $(x, y, z)$ solution of $(\mathcal{F}(a)),(x, y, z, 0)$ is a solution of $(\mathcal{G}(a))$; if $a=0$, for every $(x, y, z)$ solution of $(\mathcal{F}(-1)),(x, y, z, 1)$ is a solution of $(\mathcal{G}(a))$.

(B) Suppose $m / d$ odd. From Proposition 2.9, $N_{3}(a)>0 \Leftrightarrow m>d$. Thus $(\mathcal{F}(a))$ has a solution if and only if $m / d>1$.

\section{The numbers $v\left(p^{m}, k\right)$}

Proposition 3.1. We have $v\left(p^{m}, k\right) \geq 3$. Moreover, if $m$ divides $2 r$, then $v\left(2^{m}, k\right)=\infty$.

Proof. Similar to the proof of Proposition 3.1 in [3].

Proposition 3.2. (1) If $m / d \notin\{1,2,4\}$, then $v\left(p^{m}, k\right)=3$.

(2) If $m / d=4$, then $v\left(p^{m}, k\right)=4$.

Proof. If $m / d \notin\{1,2,4\}$, Corollary 2.10 implies the existence of $\left(a_{1}, a_{2}, a_{3}\right) \in$ $F^{3}$ solution of $(\mathcal{F}(0))$. If $m / d=4$, Corollary 2.10 implies the existence of $\left(a_{1}, a_{2}, a_{3}, a_{4}\right) \in F^{4}$ solution of $(\mathcal{G}(0))$. Let $\left(b_{1}, b_{2}\right) \in F^{2}$ be a solution of $\left(\mathcal{E}\left(a_{1}, a_{2}, 0,1\right)\right)$, with $(\mathcal{E})$ defined by $(2.3)$. As for the proof of Proposition 3.2 in [3], we get:

(1) If $m / d \notin\{1,2,4\}$, then

$$
\left(a_{1} T+b_{1}\right)^{k}+\left(a_{2} T+b_{2}\right)^{k}+\left(a_{3} T\right)^{k}=T+\left(b_{1}\right)^{k}+\left(b_{2}\right)^{k},
$$

so that $T$ is a sum of three $k$-th powers of linear polynomials.

(2) If $m / d=4$, then

$$
\left(a_{1} T+b_{1}\right)^{k}+\left(a_{2} T+b_{2}\right)^{k}+\left(a_{3} T\right)^{k}+\left(a_{4} T\right)^{k}=T+\left(b_{1}\right)^{k}+\left(b_{2}\right)^{k},
$$

so that $T$ is a sum of four $k$-th powers of linear polynomials.

In the first case, Proposition 3.1 gives $v\left(p^{m}, k\right)=3$. In the second case, we have $v\left(p^{m}, k\right) \leq 4$. We end the proof by proving that $v\left(p^{m}, k\right)>3$ as we did in the proof of Proposition 3.2 in [3]. 
Corollary 3.3. We have $\mathcal{S}(F, k)=F[T]$ if and only if $m / d \geq 3$. More precisely, if either, $m / d$ is odd and $m \neq d$, or, if $m / d$ is even and $m / d>4$, then every $A \in F[T]$ is sum of three $k$-th powers; if $m=4 d$, then every $A \in F[T]$ is a sum of four $k$-th powers.

We are ready to present our first result.

Proposition 3.4. Assume that $m$ does not divide $2 r$. Let

$$
\gamma(m)=\left\{\begin{array}{lll}
2 & \text { if } p^{m} \equiv 1 \quad(\bmod 4) \\
3 & \text { if } p^{m} \equiv 3 \quad(\bmod 4)
\end{array}\right.
$$

(1) Let $s \geq\left[\frac{\log k}{\log (k /(k-1))}\right]$. Then, every $P \in F[T]$ of degree $\geq \delta(s, k)=$ $k\left\lceil\frac{k^{2}-2 k-k^{2}\left(1-\frac{1}{k}\right)^{s+1}}{1-k\left(1-\frac{1}{k}\right)^{+1}}\right\rceil-k+1$ is the strict sum of $s+\gamma(m)+v\left(p^{m}, k\right)$ $k$-th powers.

Moreover, if $s \geq \frac{\log k}{\log (k /(k-1))}$, then $\delta(s, k) \leq k^{4}-3 k^{3}+2 k^{2}-2 k+1$.

(2) Let $s \geq \frac{\log (k(k-1) / 2)}{\log (k /(k-1))}$. Then, every $P \in F[T]$ of degree $\geq k^{3}-3 k+1$ is a strict sum of $s+\gamma(m)+v\left(p^{m}, k\right) k$-th powers.

(3) Let $s \geq \frac{3 \log k}{\log (k /(k-1))}-1$. Then, every $P \in F[T]$ such that $k^{3}-2 k^{2}-$ $k+1 \leq \operatorname{deg} P \leq k^{3}-3 k$ is the strict sum of $s+\gamma(m)+v\left(p^{m}, k\right) k$-th powers.

Proof. From Propositions 2.7 and $3.2, F$ is a $k$-Waring field and $v\left(p^{m}, k\right)$ is finite. Let $w(m, k)=v\left(p^{m}, k\right)+\max \left(\ell\left(p^{m}, k\right), 1+\lambda\left(p^{m}, k\right)\right)$. From [2, Proposition 5.3], we have the following facts:

(1) Let $s \geq\left[\frac{\log k}{\log (k /(k-1))}\right]$. Then every $P \in F[T]$ of degree $\geq \delta(s, k)=$ $k\left\lceil\frac{k^{2}-2 k-k^{2}\left(1-\frac{1}{k}\right)^{s+1}}{1-k\left(1-\frac{1}{k}\right)^{s+1}}\right\rceil-k+1$ is a strict sum of $\left.s+w(m, k)\right) k$-th powers. Moreover, if $s \geq \frac{\log k}{\log (k /(k-1))}$, then $\delta(s, k) \leq k^{4}-3 k^{3}+2 k^{2}-2 k+1$.

(2) Let $s \geq \frac{\log (k(k-1) / 2)}{\log (k /(k-1))}$. Then every $P \in F[T]$ of degree $\geq k^{3}-3 k+1$ is the strict sum of $s+w(m, k) k$-th powers.

(3) Let $s \geq \frac{3 \log k}{\log (k /(k-1))}-1$. Then every $P \in F[T]$ such that

$$
k^{3}-2 k^{2}-k+1 \leq \operatorname{deg} P \leq k^{3}-3 k
$$

is the strict sum of $s+w(m, k) k$-th powers.

From Proposition 2.7, $\ell\left(2^{m}, k\right)=2$. From Remark $2.8, \lambda\left(p^{m}, k\right)=1$ or 2 according as $p^{m} \equiv 1$ or $3(\bmod 4)$, so that, with $(3.1), w(m, k)=v\left(p^{m}, k\right)+$ $\gamma(m)$.

Corollary 3.5. (1) Suppose $p^{m} \equiv 1(\bmod 4)$.

(a) If either, $m / d$ is odd and $m \notin\{1, r\}$, or, if $m / d$ is even and $m / d>4$, then $G\left(p^{m}, k\right) \leq\left[\frac{\log k}{\log (k /(k-1))}\right]+5 \leq k \log k+5$.

(b) If $m / d=4$, then $G\left(p^{m}, k\right) \leq\left[\frac{\log k}{\log (k /(k-1))}\right]+6 \leq k \log k+6$.

(2) Suppose $p^{m} \equiv 3(\bmod 4)$. If $m \notin\{1, r\}$, then $G\left(p^{m}, k\right) \leq\left[\frac{\log k}{\log (k /(k-1))}\right]+$ $6 \leq k \log k+6$. 
Proof. Apply the first of part of the previous proposition.

The following proposition gives an example of an infinite sequence of polynomials which are sums of $k$-th powers and not strict sums of $k$-th powers.

Proposition 3.6. Suppose $m=2 d$. Let $a \in F$ be such that $a \notin \mathbb{F}_{q}$. Let $b \in F$ be such that $b^{Q}=a$. For $n \geq Q$, let

$$
B_{n}=a T^{n k}+b T^{n k+1-Q^{2}} .
$$

Then $B_{n}$ is a sum of three $k$-th powers and is not a strict sum of $k$-th powers.

Proof. Similar to the proof of Proposition 3.7 in [3].

Corollary 3.7. If $m / d=2$, then $G\left(p^{m}, k\right)=\infty$.

\section{Strict sums of degree $\leq k(k-2)$}

The two following propositions form the key of the proof.

Proposition 4.1. For $i \in\{0, \ldots, Q-1\}$ and $X \in F[T]$ let

$$
L_{i}(X)=X^{Q} T^{i}+X T^{Q i} .
$$

Then, the map $X \mapsto L_{i}(X)$ is additive and the following identities are satisfied:

$$
L_{i}(X)=\left(X+\frac{1}{2} T^{i}\right)^{Q+1}-\left(X-\frac{1}{2} T^{i}\right)^{Q+1} .
$$

For every $b \in F$,

$$
L_{i}\left(X+b T^{i}\right)=L_{i}(X)+\left(b^{Q}+b\right) T^{i(Q+1)} .
$$

Moreover, if $F \subset \mathbb{F}_{Q^{2}}$, then, for every $c \in F^{\times}$,

$$
L_{i}(X)+c^{Q+1} T^{(Q+1) i}=\left(\frac{1}{c^{Q}} X+c T^{i}\right)^{Q+1}-\left(\frac{1}{c^{Q}} X\right)^{Q+1}
$$

Proof. The proof of (4.2) and (4.3) is immediate. We get (4.4) from observing that $c^{Q^{2}}=c$.

Proposition 4.2. Suppose $F=\mathbb{F}_{q}$.

(1) For every $(a, b, c) \in F^{3}$, the polynomial $c+b T+b T^{Q}+a T^{Q+1}$ is a strict sum of three $k$-th powers.

(2) Let $c \in F$. There exists $\left(\alpha_{1}, \alpha_{2}, \alpha_{3}, \beta_{1}, \beta_{2}, \beta_{3}\right) \in F^{6}$ such that for $i \in$ $\{0, \ldots, Q-1\}$ and $X \in F[T]$,

(4.5) $L_{i}(X)+c T^{(Q+1) i}=\left(\alpha_{1} X+\beta_{1} T^{i}\right)^{k}+\left(\alpha_{2} X+\beta_{2} T^{i}\right)^{k}+\left(\alpha_{3} X+\beta_{3} T^{i}\right)^{k}$.

Proof. (1) Let $(a, b, c) \in F^{3}$ and let

$$
A=a+b T+b T^{Q}+c T^{Q+1} .
$$


From Lemma 2.3, there is $(x, y, z, \xi, \eta, \zeta) \in F^{6}$ such that

$$
\left\{\begin{array}{l}
a=x^{2}+y^{2}+z^{2} \\
b=x \xi+y \eta+z \zeta \\
c=\xi^{2}+\eta^{2}+\zeta^{2}
\end{array}\right.
$$

Since $F \subset \mathbb{F}_{Q}$, for every $u \in F$, we have $u^{Q}=u$, so that,

$$
\left\{\begin{array}{l}
a=x^{Q+1}+y^{Q+1}+z^{Q+1} \\
b=x^{Q} \xi+y^{Q} \eta+z^{Q} \zeta \\
c=\xi^{Q+1}+\eta^{Q+1}+\zeta^{Q+1}
\end{array}\right.
$$

Hence,

$$
a+b T+b T^{Q}+c T^{Q+1}=(x+\xi T)^{Q+1}+(y+\eta T)^{Q+1}+(z+\zeta T)^{Q+1},
$$

so that $A$ is a strict sum of three $k$-th powers.

(2) Apply (†) with $a=0, b=1$. There exists $\left(\alpha_{1}, \alpha_{2}, \alpha_{3}, \beta_{1}, \beta_{2}, \beta_{3}\right) \in F^{6}$ such that

$$
\left\{\begin{array}{l}
\alpha_{1}^{k}+\alpha_{2}^{k}+\alpha_{3}^{k}=0 \\
\alpha_{1}^{Q} \beta_{1}+\alpha_{2}^{Q} \beta_{2}+\alpha_{3}^{Q} \beta_{3}=\alpha_{1} \beta_{1}^{Q}+\alpha_{2} \beta_{2}^{Q}+\alpha_{3} \beta_{3}^{Q}=1 \\
\beta_{1}^{k}+\beta_{2}^{k}+\beta_{3}^{k}=c
\end{array}\right.
$$

Thus,

$\left(\alpha_{1} X+\beta_{1} T^{i}\right)^{k}+\left(\alpha_{2} X+\beta_{2} T^{i}\right)^{k}+\left(\alpha_{3} X+\beta_{3} T^{i}\right)^{k}=c T^{(Q+1) i}+X^{Q} T^{i}+X T^{Q i}$.

Proposition 4.3. Suppose that $m / d \geq 3$.

- Let $0<N<k-2$ and let

$$
A=\sum_{n=0}^{k N} a_{n} T^{n}
$$

be a polynomial of $F[T]$ such that

$$
k(N-1)<\operatorname{deg} A \leq k N .
$$

Then, $A$ is a strict sum of $k$-th powers if and only if $a_{n}=0$ for each $n \in \bigcup_{i=0}^{N-1}[i Q+N+1,(i+1) Q-1]$. Thus, $\mathcal{S}(F, k) \neq \mathcal{S}^{\times}(F, k)$ and $g\left(p^{m}, k\right)=\infty$.

- Let $A \in F[T]$ be such that

$$
k(k-3)<\operatorname{deg} A \leq k(k-2) .
$$

Then, $A$ is a strict sum of $k$-th powers.

- Let $A \in F[T]$ of degree $\leq k(k-2)$ be a strict sum of $k$-th powers. Then, $A$ is a strict sum of $v\left(p^{m}, k\right)\left\lceil\frac{\operatorname{deg} A}{k}\right\rceil+2 k$-th powers of polynomials of degree $\leq k-2$. 
- Let $A \in F[T]$ of degree $\leq k(k-2)$. Then,

$$
A=\sum_{i=1}^{s}\left(X_{i}\right)^{k}
$$

with $s=v\left(2^{m}, k\right)(k-2)+2$ and $\operatorname{deg} X_{i} \leq k-2$ for $i=1, \ldots, s$.

Proof. The proof is similar to that of Proposition 4.3 in [3]. It makes use of Lemma 2.2 and Corollary 2.10 as the proof of Proposition 4.3 in [3] makes use of Lemma 2.2 and Corollary 2.10 in [3].

Lemma 4.4. Suppose $F \subset \mathbb{F}_{Q^{2}}$. Let $A \in F[T]$ be a sum of $k$-th powers. Then $T^{Q^{2}}-T$ divides $A^{Q}-A$.

Proof. As for Lemma 4.4 in [3].

Proposition 4.5. Suppose $F \subset \mathbb{F}_{Q^{2}}$. Let

$$
A=\sum_{n=0}^{Q^{2}-1} a_{n} T^{n}
$$

be a polynomial of $F[T]$ with $\operatorname{deg} A<Q^{2}$ and such that $T^{Q^{2}}-T$ divides $A^{Q}-A$.

- For every $n=Q j+i$ with $0 \leq j<Q, 0 \leq i<Q$, one has

$$
a_{n}=\left(a_{\bar{n}}\right)^{Q} \text {, }
$$

where $\bar{n}=Q i+j$.

- For every $n=k j$ with $0 \leq j \leq Q-1, a_{n} \in F \cap \mathbb{F}_{Q}$.

- If $\operatorname{deg} A \leq Q+1$, then $A$ is a strict sum of three $k$-th powers.

- (A) If $F \subset \mathbb{F}_{Q}$ and $Q+1<\operatorname{deg} A<Q^{2}$, then $A$ is a strict sum of $3 k-6 k$-th powers.

(B) If $F \not \subset \mathbb{F}_{Q}$ and $Q+1<\operatorname{deg} A<Q^{2}$, then $A$ is a strict sum of $2 k-3$ $k$-th powers (If, in addition, $k$ divides $\operatorname{deg} A$, then $a_{\operatorname{deg} A} \in \mathbb{F}_{q}$ ).

Proof. Making use of Lemma 4.4, the proof of the first part is similar to that of Proposition 4.5-(I) in [3]. Let $n=k j$ with $0 \leq j \leq Q-1$. Then $\bar{n}=n$, so that $a_{n} \in F_{Q}$. Let $0 \leq i, j<Q$ and let $n=Q j+i \leq Q^{2}-2$ be non divisible by $Q+1$. Then

$$
a_{n} T^{n}+a_{\bar{n}} T^{\bar{n}}=L_{i}\left(a_{Q i+j} T^{j}\right)=L_{j}\left(a_{Q j+i} T^{i}\right),
$$

so that,

$$
\begin{aligned}
A & =\sum_{i=0}^{Q-1} a_{(Q+1) i} T^{(Q+1) i}+\sum_{i=0}^{Q-2} \sum_{j=i+1}^{Q-1} L_{i}\left(a_{Q i+j} T^{j}\right) \\
& =\sum_{i=0}^{Q-1} a_{(Q+1) i} T^{(Q+1) i}+\sum_{j=1}^{Q-1} \sum_{i=0}^{j-1} L_{j}\left(a_{Q j+i} T^{i}\right) .
\end{aligned}
$$


(A) Suppose $F \subset \mathbb{F}_{Q}$, that is $F=\mathbb{F}_{q}$. Firstly, we suppose $\operatorname{deg} A \leq Q+1$. Then,

$$
A=a+b T+b T^{Q}+c T^{Q+1}
$$

with $a, b, c \in F$. From Proposition $4.2, A$ is a strict sum of three $k$-th powers. This proves the second part.

Now, we suppose $Q+1<\operatorname{deg} A \leq Q^{2}-1$. By (1),

$$
A=a_{0}+L_{1}\left(a_{Q}\right)+a_{Q+1} T^{Q+1}+\sum_{j=2}^{Q-1}\left(a_{(Q+1) j} T^{(Q+1) j}+L_{j}\left(B_{j}\right)\right)
$$

with

$$
B_{j}=\sum_{i=0}^{j-1} a_{Q j+i} T^{i}
$$

From Proposition 4.2 , for every $j=2, \ldots, Q-1$, there exist $\left(\alpha_{j, 1}, \alpha_{j, 2}, \alpha_{j, 3}\right.$, $\left.\beta_{j, 1}, \beta_{j, 2}, \beta_{j, 3}\right) \in F^{6}$ such that

$$
a_{(Q+1) j} T^{(Q+1) j}+L_{j}\left(B_{j}\right)=\sum_{\nu=1}^{3}\left(\alpha_{j, \nu} B_{j}+\beta_{j, \nu}\right)^{k} .
$$

Thus, $B=A-\left(a_{0}+L_{1}\left(a_{Q}\right)+a_{Q+1} T^{Q+1}\right)$ is a sum of $3(Q-2) k$-th powers. From Lemma $4.4, B^{Q}-B$ is divisible by $T^{Q^{2}}-T$, so that, $\left(a_{0}+L_{1}\left(a_{Q}\right)+\right.$ $\left.a_{Q+1} T^{Q+1}\right)^{Q}-\left(a_{0}+L_{1}\left(a_{Q}\right)+a_{Q+1} T^{Q+1}\right)$ is divisible by $T^{Q^{2}}-T$. Since $\operatorname{deg}\left(a_{0}+L_{1}\left(a_{Q}\right)+a_{Q+1} T^{Q+1}\right) \leq Q+1, a_{0}+L_{1}\left(a_{Q}\right)+a_{Q+1} T^{Q+1}$ is a strict sum of three $k$-th powers. Thus, by (3), $A$ is a sum of $3+3(Q-2) k$-th powers.

We consider the degrees. Suppose that

$$
\operatorname{deg} A=(Q+1) N-\rho \text {. }
$$

with

$$
0 \leq \rho \leq Q
$$

Observe that

$$
N<Q
$$

Let $j \in\{2, \ldots, Q-1\}$ be such that $j>N$. Then, $(Q+1) j>(Q+1) N-\rho$, so that $a_{(Q+1) j}=0$. We have $Q j+i \geq(Q+1) N+Q-N+i>(Q+1) N-\rho$, so that $a_{Q j+i}=0$. Hence, $B_{j}=0$. Thus, the $\left(\alpha_{j, \nu} B_{j}+\beta_{j, \nu}\right)$ occurring in (3) are zero polynomials. If $2 \leq j \leq N$, then by (2), $\operatorname{deg} B_{j} \leq N$. Thus, the sum (3) is a strict one.

(B) Suppose $F \not \subset \mathbb{F}_{Q}$. Since $F \subset \mathbb{F}_{Q^{2}}$, we have $F=\mathbb{F}_{q^{2}}$. Thus, $m=2 d$ and $r / d$ is odd. For every $j=0, \ldots, Q-1, a_{(Q+1) j} \in F \cap \mathbb{F}_{Q}=\mathbb{F}_{q}$. Thus, if $k=Q+1$ divides $\operatorname{deg} A$, then $a_{\operatorname{deg} A} \in \mathbb{F}_{q}$. The trace map $x \mapsto x^{q}+x$ from $F=\mathbb{F}_{q^{2}}$ to $\mathbb{F}_{q}$ is onto. For every $j=0, \ldots, Q-1$, there is $b_{j} \in F$ such that

$$
a_{(Q+1) j}=b_{j}^{q}+b_{j} .
$$


For every $y \in \mathbb{F}_{q^{2}}$, we have $y^{q^{2}}=y$, so that, by induction, for every positive integer $s$, we have $y^{q^{2 s}}=y$ and $y^{q^{2 s+1}}=y^{q}$. Since $Q=q^{r / d}$ with $r / d$ odd, for every $j=0, \ldots, Q-1$, we have

$$
a_{(Q+1) j}=b_{j}^{Q}+b_{j} .
$$

Moreover, from Proposition 2.4, each $x \in \mathbb{F}_{q}$ is a $(q+1)$-th power, so that there is $c_{j} \in F$ such that $a_{(Q+1) j}=\left(c_{j}\right)^{k}=\left(c_{j}\right)^{Q+1}$.

Suppose $\operatorname{deg} A \leq Q+1$. Then

$$
A=b_{0}+b_{0}^{Q}+L_{0}\left(a_{1} T\right)+\left(c_{1} T\right)^{Q+1} .
$$

By (4.3),

then by (4.2),

$$
A=L_{0}\left(a_{1} T+b_{0}\right)+\left(c_{1} T\right)^{Q+1},
$$

$$
A=\left(a_{1} T+b_{0}+\frac{1}{2}\right)^{Q+1}-\left(a_{1} T+b_{0}-\frac{1}{2}\right)^{Q+1}+\left(c_{1} T\right)^{Q+1} .
$$

Suppose $\operatorname{deg} A>Q+1$. Then,

$$
\begin{aligned}
A & =c_{0}^{Q+1}+\sum_{j=1}^{Q-1}\left(\left(b_{j}^{Q}+b_{j}\right) T^{j(Q+1)}+\sum_{i=0}^{j-1} L_{j}\left(a_{Q j+i} T^{i}\right)\right) \\
& =c_{0}^{Q+1}+\sum_{j=1}^{Q-1}\left(\left(b_{j}^{Q}+b_{j}\right) T^{j(Q+1)}+L_{j}\left(B_{j}\right)\right),
\end{aligned}
$$

with $B_{j}$ defined by (2). By (4.3),

$$
A=c_{0}^{Q+1}+\sum_{j=1}^{Q-1} L_{j}\left(B_{j}+b_{j} T^{j}\right)
$$

Then, by (4.2),

$$
A=c_{0}^{k}+\sum_{j=1}^{Q-1}\left(\left(B_{j}+b_{j} T^{j}+\frac{1}{2} T^{j}\right)^{k}-\left(B_{j}+b_{j} T^{j}-\frac{1}{2} T^{j}\right)^{k}\right) .
$$

From Remark $2.8,-1$ is a $k$-th power, so that $(7)$ is a sum of three $k$-th powers and $(8)$ is a sum of $(1+2(Q-1)) k$-th powers. We observe that $(7)$ is a strict sum and we finish the proof, proving as above that (8) is a strict sum.

\section{The descent process}

In this section, we use the descent process already used in [3] and [4].

Proposition 5.1. Suppose $F \subset \mathbb{F}_{Q^{2}}$. Then $\mathcal{S}(F, k)$ is the subset of $F[T]$ formed by the polynomials $A$ such that $T^{Q^{2}}-T$ divides $A^{Q}-A$.

Proof. The proof is similar to those of Proposition 5.1 and Corollary 5.2 in $[3]$. 
Lemma 5.2. Let $n$ be a positive integer and let $H \in F[T]$ be such that

$$
k(n-1)<\operatorname{deg} H \leq k n .
$$

In addition, in the case when $m=2 d$ and $\operatorname{deg} H=k n$, we suppose that the leading coefficient of $H$ is a $k$-th power. Then, we have

$$
H=\sum_{i=1}^{1+\lambda} B_{i}^{k}+\sum_{i=0}^{Q-1} L_{i}\left(Y_{i}\right)+R,
$$

with $\lambda=\lambda\left(p^{m}, k\right)$ and where $B_{1}, \ldots, B_{\lambda+1}, Y_{0}, \ldots, Y_{Q-1}, R \in F[T]$ with

$$
\begin{gathered}
\operatorname{deg} B_{1}, \ldots, \operatorname{deg} B_{\lambda+1} \leq n, \\
\operatorname{deg} Y_{0}, \ldots, \operatorname{deg} Y_{Q-1}<n, \\
\operatorname{deg} R<Q^{2}, \\
R=\sum_{i=0}^{Q-1} \sum_{j=0}^{i} x_{Q j+i} T^{Q j+i},
\end{gathered}
$$

with $x_{Q j+i} \in F$ for all $i$ and $j$. Moreover, if $\lambda\left(p^{m}, k\right)=2$ and $\operatorname{deg} H=k n$, or if $m=2 d$ and $\operatorname{deg} H=k n$, then $B_{1}=0$.

Proof. (I) Suppose $m \neq 2 d$. From Proposition $2.7, F$ is a $k$-Waring field with $\ell\left(p^{m}, k\right)=2$, so that $\max \left(\ell\left(p^{m}, k\right)-1, \lambda\left(p^{m}, k\right)\right)=\lambda\left(p^{m}, k\right)=\lambda$. From $[2$, Lemma 5.1], there exist $B_{1}, \ldots, B_{\lambda}, P \in F[T]$ such that

$$
H=B_{1}^{k}+\cdots+B_{\lambda}^{k}+P \text {, }
$$

with

$$
\operatorname{deg} B_{1}, \ldots, \operatorname{deg} B_{\lambda} \leq n, \quad \operatorname{deg} P=k n,
$$

the leading coefficient of $P$ being a $k$-th power. Observe that in the case when $\operatorname{deg} H=k n$, the leading coefficient of $H$ is a sum of two $k$-th powers, so that, when $\lambda=2$ and $\operatorname{deg} H=k n$, in (1), we can take $B_{1}=0$.

(II) Suppose $m=2 d$. From Remark $2.8,-1$ is a $k$-th power in $\mathbb{F}_{q^{2}}=F$, say $-1=b^{k}$. Thus $\lambda=1$. If $\operatorname{deg} H<k n$, then

$$
H=-T^{k n}+P,
$$

with $P$ monic of degree $k n$, so that (1) is true with $\lambda=1$ and $B_{1}=b T^{n}$. If $\operatorname{deg} H=k n$, the hypothesis insures that (1) is true with $B_{1}=0$.

Ending the proof as for Lemma 5.3 in [3], we get the identity (5.2) with degree conditions (5.3)-(5.5).

We are now ready to present our second result.

Proposition 5.3. Suppose that $m / d \geq 3$. Then:

- Every polynomial $H \in F[T]$ with degree $\geq k^{3}-2 k^{2}-k+1$ is the strict sum of $k\left(\lambda\left(p^{m}, k\right)+1\right)+v\left(p^{m}, k\right) k$-th powers. 
- Every polynomial $H \in F[T]$ with degree $\geq k^{2}-3 k+1$ is the strict sum of $(k-2) v\left(p^{m}, k\right)+k\left(\lambda\left(p^{m}, k\right)+1\right)+2 k$-th powers. Moreover, if $H \in F[T]$ is such that $k^{2}-3 k+1 \leq \operatorname{deg} H \leq k^{2}-2 k$, then $H$ is the strict sum of $(k-2) v\left(p^{m}, k\right)+2 k$-th powers.

Proof. The last claim is given by the third part of Proposition 4.3. We prove the other ones. Set $\lambda=\lambda\left(p^{m}, k\right)$. Let $H \in F[T]$ and let $n$ be the integer such that

$$
k(n-1)<\operatorname{deg} H \leq k n .
$$

From Lemma 5.2,

$$
H=\sum_{i=1}^{1+\lambda} B_{i}^{k}+\sum_{i=0}^{Q-1} L_{i}\left(Y_{i}\right)+R,
$$

where $B_{1}, \ldots, B_{1+\lambda}, Y_{0}, \ldots, Y_{Q-1}, R \in F[T]$ with

$$
\operatorname{deg} B_{1}, \ldots, \operatorname{deg} B_{1+\lambda} \leq n,
$$

$$
\operatorname{deg} Y_{0}, \ldots, \operatorname{deg} Y_{Q-1}<n,
$$

$$
\operatorname{deg} R<Q^{2} \text {. }
$$

By (4.2),

$$
L_{i}\left(Y_{i}\right)=\left(Y_{i}+\frac{1}{2} T^{i}\right)^{k}-\left(Y_{i}-\frac{1}{2} T^{i}\right)^{k} .
$$

Since -1 is a sum of $\lambda k$-th powers, for each index $i=0, \ldots, Q-1$, there is $Z_{i, 1}, \ldots, Z_{i, 1+\lambda} \in F[T]$, such that

$$
L_{i}\left(Y_{i}\right)=\left(Z_{i, 1}\right)^{k}+\left(Z_{i, 2}\right)^{k}+\cdots+\left(Z_{i, 1+\lambda}\right)^{k},
$$

and such that

$$
\operatorname{deg} Z_{i, j} \leq \max (i, n-1)
$$

Set $v=v\left(p^{m}, k\right)$. Then, there exist $a_{1}, b_{1}, \ldots, a_{v}, b_{v}$ in $F$ such that

$$
R=\left(a_{1} R+b_{1}\right)^{k}+\cdots+\left(a_{v} R+b_{v}\right)^{k} .
$$

By (2), (6) and (8),

$$
\begin{aligned}
H= & \sum_{i=1}^{1+\lambda} B_{i}^{k}+\sum_{i=0}^{Q-1}\left(\left(Z_{i, 1}\right)^{k}+\cdots+\left(Z_{i, 1+\lambda}\right)^{k}\right) \\
& +\left(a_{1} R+b_{1}\right)^{k}+\cdots+\left(a_{v} R+b_{v}\right)^{k}
\end{aligned}
$$

so that $H$ is a sum of $((\lambda+1)(Q+1)+v) k$-th powers of polynomials. By $(3),(4),(5),(7)$ and $(8)$, these polynomials have their degrees bounded by $\max \left(n, Q^{2}-1\right)$. In view of (1), if $n \geq Q^{2}-1$, the above sum is a strict one. This proves the first part. 
We have $\operatorname{deg} R<Q^{2}$. From the fourth part of Proposition 4.3, $R$ is a sum of

$$
s=(k-2) v\left(p^{m}, k\right)+2
$$

$k$-th powers of polynomials of degree $\leq Q-1$. Thus by (2) and (6), $H$ is a sum of

$$
k(\lambda+1)+s=(k-2) v\left(p^{m}, k\right)+k(\lambda+1)+2
$$

$k$-th powers of polynomials of degree $\leq \max (n, Q-1)$. In view of (1), if $n \geq Q-1$, the sum is a strict representation. This proves the second part.

Corollary 5.4. Suppose that $m / d \geq 3$. Then,

$$
\mathcal{S}^{\times}\left(p^{m}, k\right)=\mathcal{A}_{\infty} \cup\left(\bigcup_{N=0}^{k-3} \mathcal{A}_{N}\right),
$$

where

$$
\begin{aligned}
\mathcal{A}_{\infty} & =\{A \in F[T] \mid \operatorname{deg} A>k(k-3)\}, \\
\mathcal{A}_{0} & =F
\end{aligned}
$$

and for $N=1, \ldots, k-3$,

$$
\mathcal{A}_{N}=\left\{A \in F[T] \mid A=\sum_{n=0}^{N} \sum_{i=0}^{N} x_{n, i} T^{i+n Q}\right\}
$$

with $x_{n, i} \in F$. Moreover,

(1) if $p^{m}$ is congruent to 1 modulo 4 and $m / d \neq 4$,

$$
G\left(p^{m}, k\right)=G^{\times}\left(p^{m}, k\right) \leq 2 k+3 ;
$$

(2) if $p^{m}$ is congruent to 3 modulo 4 ,

$$
G\left(p^{m}, k\right)=G^{\times}\left(p^{m}, k\right) \leq 3 k+3 ;
$$

(3) if $m / d=4$,

$$
G\left(p^{m}, k\right)=G^{\times}\left(p^{m}, k\right) \leq 2 k+4 ;
$$

(4) if $p^{m}$ is congruent to 1 modulo 4 and $m / d \neq 4$,

$$
g\left(p^{m}, k\right)=\infty, \quad g^{\times}\left(p^{m}, k\right) \leq 5 k-4 ;
$$

(5) if $p^{m}$ is congruent to 3 modulo 4 ,

$$
g\left(p^{m}, k\right)=\infty, \quad g^{\times}\left(p^{m}, k\right) \leq 6 k-4 ;
$$

(6) if $m / d=4$,

$$
g\left(p^{m}, k\right)=\infty, \quad g^{\times}\left(p^{m}, k\right) \leq 6 k-6 .
$$


Proof. The first assertion is given by Propositions 4.3 and 5.3. From Corollary $3.3, \mathcal{S}^{\times}\left(p^{m}, k\right) \neq \mathcal{S}\left(p^{m}, k\right)$, so that $g\left(p^{m}, k\right)=\infty$. The bounds for $G\left(p^{m}, k\right)$ are obtained by noting that from Proposition $2.7, \lambda\left(p^{m}, k\right) \in\{1,2\}$ and from Remark 2.8, $\lambda\left(p^{m}, k\right)=1$ when $p^{m}$ is congruent to 1 modulo 4 . We deduce from Propositions 4.3 and 5.3 that

$$
g^{\times}\left(p^{m}, k\right) \leq(k-2) v\left(p^{m}, k\right)+k\left(\lambda\left(p^{m}, k\right)+1\right)+2 .
$$

Bounds for $g^{\times}\left(p^{m}, k\right)$ in parts $4-6$ are given by Propositions 3.4, 4.3 and 5.3 .

Remark 5.5. If $k \geq 20$, or if $k=18,14$, for all $m$, the bounds for the numbers $G\left(p^{m}, k\right)$ given by this corollary are better than those given by Corollary 3.5 ; if $k=4,6$, the old bounds are better in all cases. If $k=12,10,8$, the new bounds are better when $m / d$ is even.

Proposition 5.6. Suppose $m / d \leq 2$.

(A) (a) If $m=d$ and if $p^{m}$ is congruent to 1 modulo 4 , every $H \in \mathcal{S}(F, k)$ with degree $\geq k^{2}-3 k+1$ is a strict sum of $2 k k$-th powers.

(b) If $m=d$ and if $p^{m}$ is congruent to 3 modulo 4 , every $H \in \mathcal{S}(F, k)$ with degree multiple of $k$ is a strict sum of $3 k-1 k$-th powers; every $H \in \mathcal{S}(F, k)$ with degree non multiple of $k$ is a strict sum of $3 k k$-th powers.

(B) If $m=2 d$, every $H \in \mathcal{S}(F, k)$ with degree multiple of $k$ and whose leading coefficient is a $k$-th power in the field $F$ is a strict sum of $2 k-1 k$-th powers; every $H \in \mathcal{S}(F, k)$ of degree non multiple of $k$ is a strict sum of $2 k k$-th powers.

Proof. Let $H \in \mathcal{S}(F, k)$ be such that

$$
k(n-1)<\operatorname{deg} H \leq k n .
$$

In addition, in the case when $m=2 d$ and $\operatorname{deg} H=k n$, we suppose that the leading coefficient of $H$ is a $k$-th power.

We have

$$
H=\sum_{i=1}^{1+\lambda} B_{i}^{k}+\sum_{i=0}^{Q-1} L_{i}\left(Y_{i}\right)+R
$$

where $B_{1}, \ldots, B_{1+\lambda}, Y_{0}, \ldots, Y_{Q-1}, R \in F[T]$ are as in Lemma 5.2 , so that

$$
R=\sum_{i=0}^{Q-1} \sum_{j=0}^{i} x_{Q j+i} T^{Q j+i} .
$$

In view of $(4.2), H-R$ is a sum of $k$-th powers. Since $H \in \mathcal{S}(F, k), R$ is also a sum of $k$-th powers. From (3), Lemma 4.4 and Proposition 4.5, if 
$n \in\left\{0, \ldots, Q^{2}-1\right\}$ is not a multiple of $Q+1$, then $x_{n}=0$; if $n \in\left\{0, \ldots, Q^{2}-1\right\}$ is a multiple of $Q+1=k$, then $x_{n} \in F \cap \mathbb{F}_{Q}=\mathbb{F}_{q}$. Thus,

$$
H=\sum_{i=1}^{1+\lambda} B_{i}^{k}+\sum_{i=0}^{Q-1}\left(L_{i}\left(Y_{i}\right)+x_{k i} T^{k i}\right),
$$

with

$$
x_{k i} \in \mathbb{F}_{q} \text { for } \quad 0 \leq i \leq Q-1 .
$$

(A) Suppose that $m$ divides $r$ so that $F=\mathbb{F}_{q}$. From (4.2) or (4.4), every $L_{i}\left(Y_{i}\right)+x_{k i} T^{k i}$ in (4) is a sum of $1+\lambda k$-th powers. By (2), (5.3), (5.4), (3) and (4), $H$ is a sum of $(\theta(q, H)+(1+\lambda) Q) k$-th powers of polynomials of degree $\leq \mu=\max (n, Q-1)$ with

$$
\theta(q, H)=\left\{\begin{array}{ccc}
2 & \text { if } \operatorname{deg} H=k n, \\
2 & \text { if } \operatorname{deg} H<k n & \text { and } \quad q \equiv 1 \quad(\bmod 4), \\
3 & \text { if } \operatorname{deg} H<k n & \text { and } \quad q \equiv 3 \quad(\bmod 4)
\end{array}\right.
$$

In view of (1), if $n \geq Q-1$, the sum is a strict one. Suppose $p^{m} \equiv$ $3(\bmod 4)$. If $n<Q-1$, then $\operatorname{deg} H<Q^{2}-1$. From Proposition 4.5, $H$ is a strict sum of $3 k-6 \leq(\theta(q, H)+(1+\lambda) Q) k$-th powers.

(B) Suppose $m=2 d$. In this case, -1 is a $k$-th power in $F, \mathbb{F}_{q^{2}} \subset F$ and $x_{k i} \in \mathbb{F}_{q}$ for each $i=0, \ldots, Q-1$, so that, for each $i=0, \ldots, Q-1$, there exists $y_{i} \in \mathbb{F}_{q^{2}}$ such that $x_{k i}=y_{i}^{k}$. From (4.2) or (4.4), $L_{i}\left(Y_{i}\right)+\left(x_{k i}\right) T^{k i}=$ $L_{i}\left(Y_{i}\right)+\left(y_{k i}\right)^{Q+1} T^{k i}$ is a sum of two $k$-th powers. Moreover, if $\operatorname{deg} H=k n$, then, in (4) we have $B_{1}=0$, so that $H$ is a sum of $(\eta(H)+2 Q) k$-th powers, where

$$
\eta(H)=\left\{\begin{array}{lll}
1 & \text { if } \quad \operatorname{deg} H=k n \\
2 & \text { if } \quad \operatorname{deg} H<k n
\end{array}\right.
$$

As above, if $n \geq Q-1$, the sum is strict. In the case when $n<Q-1$ we conclude with Proposition 4.5.

Corollary 5.7. $\quad$ Suppose that $m$ divides $r$. Then,

$$
\mathcal{S}^{\times}\left(p^{m}, k\right)=\mathcal{S}\left(p^{m}, k\right)=\left\{A \in F[T] \mid A^{Q}-A \equiv 0 \quad\left(\bmod \quad T^{Q^{2}}-T\right)\right\} .
$$

Moreover,

(1) if $p^{m}$ is congruent to 1 modulo 4 ,

$$
\begin{aligned}
G\left(p^{m}, k\right) & =G^{\times}\left(p^{m}, k\right) \leq 2 k, \\
g\left(p^{m}, k\right) & =g^{\times}\left(p^{m}, k\right) \leq 3 k-6 ;
\end{aligned}
$$

(2) if $p^{m}$ is congruent to 3 modulo 4 ,

$$
\begin{aligned}
G\left(p^{m}, k\right) & =G^{\times}\left(p^{m}, k\right) \leq 3 k, \\
g\left(p^{m}, k\right) & =g^{\times}\left(p^{m}, k\right) \leq 3 k .
\end{aligned}
$$


- Suppose that $m / d=2$. Then,

$$
\mathcal{S}\left(p^{m}, k\right)=\left\{A \in F[T] \mid A^{Q}-A \equiv 0 \quad\left(\bmod \quad T^{Q^{2}}-T\right)\right\},
$$

$\mathcal{S}^{\times}\left(p^{m}, k\right)$ is the set of $A \in \mathcal{S}\left(p^{m}, k\right)$ such that either $\operatorname{deg} A$ is not a multiple of $k$, or $\operatorname{deg} A$ is a multiple of $k$ and the leading coefficient of $A$ is in the field $\mathbb{F}_{q}$. Moreover, we have

$$
G\left(p^{m}, k\right)=g\left(p^{m}, k\right)=\infty, \quad G^{\times}\left(p^{m}, k\right) \leq g^{\times}\left(p^{m}, k\right) \leq 2 k .
$$

Proof. With Propositions 4.3, 4.5, Propositions 5.1, 5.3 and 5.5. In the case where $m$ divides $r$ and $p^{m}$ is congruent to 1 modulo 4 , we get

$$
g\left(p^{m}, k\right)=g^{\times}\left(p^{m}, k\right) \leq \max (2 k, 3 k-6) .
$$

Observe that $\max (2 k, 3 k-6)=3 k-6$ since $2 k>3 k-6$ implies $k=4, p=$ $3, m=1$, a contradiction.

Remark 5.8. (1) In the case $k=4$, we have $p=3, r=d=1$. Corollaries 3.5 and 5.4 give $G\left(3^{m}, 4\right)=G^{\times}\left(3^{m}, 4\right) \leq 9$ for even $m>4$ and $G\left(3^{m}, 4\right)=$ $G^{\times}\left(3^{m}, 4\right) \leq 10$ for odd $m>1$ or for $m=4$. These bounds were proved in [4]. It also gives $g^{\times}\left(3^{m}, 4\right) \leq 16$ for even $m>4, g^{\times}\left(3^{m}, 4\right) \leq 20$ for odd $m>1$ and $g^{\times}(81,4) \leq 18$. In the case of even $m$, this improves the bounds obtained in [4].

(2) In the case $k=4$, Corollary 5.6 gives the following bounds:

$$
\begin{array}{cl}
G(3,4)=G^{\times}(3,4) \leq 12, & g(3,4)=g^{\times}(3,4) \leq 12 ; \\
G(9,4)=\infty, \quad G^{\times}(9,4) \leq 8, & g(9,4) \leq \infty, \quad g^{\times}(9,4) \leq 8 ;
\end{array}
$$

which are the bounds given in [4].

(3) For $k=p^{r}+1$ tending to $\infty$, we have $G^{\times}\left(p^{m}, k\right)<<k$ as well as $g^{\times}\left(p^{m}, k\right)<<k$ unlike to the classical Waring numbers $G_{\mathbb{N}}(k)$ and $g_{\mathbb{N}}(k)$. Indeed, from [7], $g_{\mathbb{N}}(k)>>2^{k}$ when from [23], $G_{\mathbb{N}}(k)<<k \log k$.

Acknowledgements. I thank an unknown referee for useful remarks, specially for the information concerning the existence of De Konninck and A. Mercier's book

\section{References}

[1] N. Bourbaki, Éléments de mathématique, Fasc. XXIII, Livre II: Algébre. Chapitre 8: Modules et anneaux semi-simples. Nouveau tirage de l'édition de 1958. Actualités Scientifiques et Industrielles, No. 1261. Hermann, Paris, 1973.

[2] M. Car, New bounds on some parameters in the Waring problem for polynomials over a finite field, Finite fields and applications, 59-77, Contemp. Math., 461, Amer. Math. Soc., Providence, RI, 2008.

[3] two, Port. Math., to appear.

[4] - Sums of fourth powers of polynomials over a finite field of characteristic 3, Funct. Approx. Comment. Math. 38 (2008), part 2, 195-220.

[5] M. Car and L. Gallardo, Sums of cubes of polynomials, Acta Arith. 112 (2004), no. 1, 41-50. 
[6] G. Effinger and D. R. Hayes, Additive Number Theory of Polynomials over a Finite Field, Oxford Mathematical Monographs, Clarendon Pres, Oxford, 1991.

[7] W. J. Ellison, Waring's problem, Amer. Math. Monthly 78 (1971), no. 1, 10-36.

[8] L. H. Gallardo, On the restricted Waring problem over $\mathbb{F}_{2^{n}}[t]$, Acta Arith. 42 (2000), no. 2, 109-113.

[9] - Every strict sum of cubes in $\mathbb{F}_{4}[t]$ is a strict sum of 6 cubes, Port. Math. 65 (2008), no. 2, 227-236.

[10] L. H. Gallardo and D. R. Heath-Brown, Every sum of cubes in $\mathbb{F}_{2}[t]$ is a strict sum of 6 cubes, Finite Fields Appl. 13 (2007), no. 4, 981-987.

[11] L. H. Gallardo and L. N. Vaserstein, The strict Waring problem for polynomials rings, J. Number Theory 128 (2008), no. 12, 2963-2972.

[12] G. H. Hardy and E. M. Wright, An Introduction to the Theory of Numbers, Oxford University Press, 4th. Ed., 1960.

[13] J.-M. De Konninck and A. Mercier, 1001 problems in classical number theory, Trans. from the French by J.-M. De Konninck, (English), Providence, RI: (AMS) xii, (2007).

[14] R. M. Kubota, Waring's problem for $\mathbb{F}_{q}[x]$, Dissertationes Math. (Rozprawy Mat.) 117 (1974), 60 pp.

[15] R. E. A. C. Paley, Theorems on polynomials in a Galois field, The Quarterly Journal of Mathematics. 4 (1933), 52-63.

[16] C. Small, Sums of powers in large finite fields, Proc. Amer. Math. Soc. 65 (1977), no. 1, 35-36.

[17] L. N. Vaserstein, Waring's problem for algebras over fields, J. Number Theory 26 (1987), no. 3, 286-298.

[18] Waring's problem for commutative rings, J. Number Theory 26 (1987), no. 3, 299-307.

[19] _ Sums of cubes in polynomial rings, Math. Comput. 56 (1991), no. 193, 349-357.

[20] _ Ramsey's theorem and Waring's problem for algebras over fields, The arithmetic of function fields (Columbus, OH, 1991), 435-441, Ohio State Univ. Math. Res. Inst. Publ., 2, de Gruyter, Berlin, 1992.

[21] Y.-R. Yu and T. Wooley, The unrestricted variant of Waring's problem in function fields, Funct. Approx. Comment. Math. 37 (2007), part 2, 285-291.

[22] W. A. Webb, Waring's problem in $G F[q, x]$, Acta Arith. 22 (1973), 207-220.

[23] T. Wooley, Large improvements in Waring's problem, Ann. of Math. (2) 135 (1992), no. $1,131-164$

\section{LATP-UMR CNRS 6632}

Amu, Case Cour A

Avenue Escadrille Normandie-Niemen

F-13397 Marseille Cedex 20, France

E-mail address: mireille.car@univ-amu.fr 